\title{
Urinary oxytocin positively correlates with performance in facial visual search in unmarried males, without specific reaction to infant face
}

\section{Atsuko Saito ${ }^{1 *}$, Hiroki Hamada ${ }^{2}$, Takefumi Kikusui ${ }^{3}$, Kazutaka Mogi ${ }^{3}$, Miho Nagasawa ${ }^{3}$, Shohei Mitsui ${ }^{3}$, Takashi Higuchi ${ }^{4}$, Toshikazu Hasegawa ${ }^{1}$ and Kazuo Hiraki ${ }^{2,5 *}$}

1 Department of Cognitive and Behavioral Science, Graduate School of Arts and Sciences, The University of Tokyo, Tokyo, Japan

2 Department of General Systems Studies, Graduate School of Arts and Sciences, The University of Tokyo, Tokyo, Japan

${ }^{3}$ Department of Animal Science and Biotechnology, Azabu University, Kanagawa, Japan

${ }^{4}$ Faculty of Medical Sciences, University of Fukui, Fukui, Japan

${ }^{5}$ JST, CREST, Tokyo, Japan

\section{Edited by:}

Tomoko Soga, Monash University

Sunway Campus, Malaysia

Reviewed by:

Hiroyuki Minakata, Suntory Institute

for Bioorganic Research, Japan

Dirk Scheele, University of Bonn,

Germany

${ }^{*}$ Correspondence:

Atsuko Saito, Department of Cognitive and Behavioral Science,

Graduate School of Arts and

Sciences, The University of Tokyo,

3-8-1 Komaba, Meguro-ku, Tokyo

153-8902, Japan

e-mail: casaito@mail.ecc.u-tokyo.

ac.jp;

Kazuo Hiraki, Department of General

Systems Studies, Graduate School

of Arts and Sciences, The University

of Tokyo, 3-8-1 Komaba, Meguro-ku,

Tokyo 153-8902, Japan

e-mail:khiraki@idea.c.u-tokyo.ac.jp
The neuropeptide oxytocin plays a central role in prosocial and parental behavior in non-human mammals as well as humans. It has been suggested that oxytocin may affect visual processing of infant faces and emotional reaction to infants. Healthy male volunteers $(N=13)$ were tested for their ability to detect infant or adult faces among adult or infant faces (facial visual search task). Urine samples were collected from all participants before the study to measure the concentration of oxytocin. Urinary oxytocin positively correlated with performance in the facial visual search task. However, task performance and its correlation with oxytocin concentration did not differ between infant faces and adult faces. Our data suggests that endogenous oxytocin is related to facial visual cognition, but does not promote infant-specific responses in unmarried men who are not fathers.

Keywords: oxytocin, social cognition, infant, visual search, cognitive task

\section{INTRODUCTION}

Oxytocin is a 9-amino-acid peptide that is produced in the hypothalamus and is released into the brain and bloodstream (Donaldson and Young, 2008). It was originally known as a hormone that increases uterus contractions during labor and stimulates the ejection of milk. In the past few decades, although controversial, current evidence suggest that oxytocin has a role to play in social and maternal behavior. Rats treated with oxytocin receptor antagonists and oxytocin knockout mice are unable to recognize previously encountered conspecifics (Engelmann et al., 1998; Ferguson et al., 2000). In prairie voles, infusion of oxytocin in the ventricle promotes pair bond formation in virgin females (Williams et al., 1994; Insel and Hulihan, 1995). Infusion of oxytocin into the ventricle can also initiate maternal behaviors in virgin female rats, such as nest building as well as licking and retrieving pups (Pedersen and Prange, 1979; Pedersen et al., 1982).

In humans, the role of oxytocin in maternal behavior and mother-infant relations, as well as in paternal behavior and father-infant relations, has been investigated. Plasma oxytocin levels in mothers positively correlated to the amount of maternal bonding behaviors, such as gaze, vocalizations, affectionate touch, mother-infant behavioral synchrony, and attachment style (Feldman et al., 2007, 2011; Strathearn et al., 2009; Gordon et al., 2010a,b). Paternal behavior and father-infant affect synchrony have also been associated with oxytocin levels in plasma and saliva (Gordon et al., 2010a,b,c; Feldman et al., 2011). Both fathers and mothers who provide high levels of contact toward their infants show increased salivary oxytocin following parent-infant interactions, and such an increase is not observed among parents displaying low levels of contact (Feldman et al., 2010). These results suggest that peripheral oxytocin levels are positively related to maternal and paternal behavior, and to parental attachment to infants in mothers and fathers.

Evidence indicating that intranasal oxytocin administration affects social perception, cognition, and behavior in a nonparental context is accumulating (Bos et al., 2012; Churchland and Winkielman, 2012; Guastella and Macleod, 2012; Van Ijzendoorn and Bakermans-Kranenburg, 2012; Zink and MeyerLindenberg, 2012). However, it is still not clear whether 
exogenously administered oxytocin, which increases oxytocin level temporally, has the same effects on social cognition or behavior as basal oxytocin levels. In fact, in the context of a trust game, administered oxytocin causes a substantial increase in trust (Kosfeld et al., 2005), but baseline plasma oxytocin levels do not associate with trust (Zak et al., 2005). In parental contexts, although some research investigated the relationship between oxytocin and perception for infants stimuli or paternal behavior by using intranasal oxytocin administration (Riem et al., 2011; Rupp et al., 2012; Weisman et al., 2012, 2014), the role of oxytocin in infant stimuli perception remains to be evaluated (Van Ijzendoorn and Bakermans-Kranenburg, 2012).

The cognitive processes associated with the perception of infant stimuli that relate to parental behavior are unknown. As mentioned above, many previous studies investigating the relationship between parental behavior and endocrinology focused on observed, direct behavior toward infants, or subjective evaluation of emotion against infants in parents (Fleming et al., 1987; Feldman et al., 2007; Gordon et al., 2010a). In addition, some neuroimaging studies have examined brain activation in response to infant stimuli (Swain, 2008), and a few researchers have reported the cognitive effects of infant stimuli (Brosch et al., 2007, 2008; Nittono et al., 2012). However, the question of how infant stimuli are processed, cognitively, remains open. Given the response to infant stimuli can be related to maltreatment of infants, it is important for researchers to understand these cognitive processes.

In rodents, the role of endocrinological factors, including oxytocin, in parental behavior has been investigated in both parental and non-parental individuals. However, in humans, it is mainly investigated in parents. Currently, we do not know whether oxytocin has the same role in non-parental humans as observed in parental individuals because parents have a different physiological status from those of non-parents. For example, fathers and mothers who have young children have lower testosterone levels than non-fathers and non-mothers (Kuzawa et al., 2010; Gettler et al., 2011). Compared to non-fathers, primate fathers that help mothers raise their young like humans have an increased density of dendritic spines on pyramidal neurons and increased vasopressin receptors in the prefrontal cortex (Kozorovitskiy et al., 2006). Therefore, the effect of oxytocin on parental behavior or response to infant stimuli in non-parents may differ from that in parents.

The purpose of this study was to investigate the role of oxytocin in non-parents in response to infant faces with comparison to adult faces. We measured urinary oxytocin levels just before the cognitive test in non-married, non-father men. We adopted the baseline peripheral oxytocin levels because nearly all previous research about oxytocin's role in parental behavior has treated them as mentioned above. We measured urinary oxytocin because it is related to social interactions (Fries et al., 2005; Nagasawa et al., 2009; Seltzer et al., 2010; Snowdon et al., 2010; Crockford et al., 2013; Wittig et al., 2014) and its sampling is non-invasive. Urinary oxytocin has a linear association with plasma oxytocin level (Amico et al., 1987; Romero et al., 2014). Women were excluded from our study to rule out possible interactions with circulating gonadal steroids (Salonia et al., 2005). Because infant faces attract human attention in the dot-probe task (Brosch et al., 2007, 2008), we measured non-parents' reaction to infants by using the attention task. The task was visual search of infant and adult faces. This task is important, requiring attention during an active scan of the visual environment, as participants search for a particular object or feature (the target) among other objects or features (distracters); it is considered a key paradigm in attention research for the investigation of selective attention (Muller and Krummenacher, 2006). If oxytocin is positively related to the responsiveness to infants, the performance for infant faces, or the performance difference between the reaction time for infant faces and that for adult faces, will negatively correlate with urinary oxytocin levels.

\section{MATERIALS AND METHODS PARTICIPANTS}

Thirteen healthy young men aged $21-33$ years $(M=26.08$, $S D=3.43$ ) were recruited from the student population of the University of Tokyo. All had normal or corrected-to-normal vision according to self-report. All were non-married and had no offspring. All protocols were approved by the Research Ethics Committee of the University of Tokyo (subject No. 229-2). Written informed consent was obtained from the participants and participants gave permission to use their data in the analyses.

\section{STIMULI AND APPARATUS}

The stimuli consisted of adult stimuli and infant stimuli. Twenty chromatic infant face photographs consisting of 10 male and 10 female Japanese infants aged from 6 to 9 months old $(M=6.5$, $S D=0.81)$ were prepared to make infant stimuli. Ten chromatic adult face photographs consisting of 6 male and 4 female Japanese adults aged $19-34$ years $(M=23.7, S D=4.43)$ and 10 photographs from the Ekman and Friesen (1976) database consisting of 4 male and 6 female Asian neutral faces were prepared to make adult stimuli. These 40 photographs were morphed using Sqirlz Morph software (Xiberpix, Solihull, UK; http://www.xiberpix. net/SqirlzMorph.html). First, 4 morphed faces were made from the following categories: adult males, adult females, infant males, and infant females. These 4 morphed faces were mixed with each original face of the same category with a mixture ratio of 1:2. All photographs were presented in gray scale, matched in brightness and contrast, and pasted onto a black background.

A 16-inch color CRT monitor attached to a PC was used to display the experimental tasks. The experimental control software was written with E-Prime 2.0 (Psychological Software Tools).

\section{PROCEDURE}

Performances were tested using a within-subjects design with 3 factors: target (present vs. absent), distracter's age category (adult vs. infant), and set size-the number of elements in the presentation. We used a variety of set sizes: 3,4 , and 6 . Each trial started with the presentation of a white fixation cross $(1.2 \times 1.2 \mathrm{~cm})$ for $500 \mathrm{~ms}$. Next, the stimulus faces were presented. The faces were $5.7 \mathrm{~cm}$ high $\times 4.5 \mathrm{~cm}$ wide on the screen, and were presented at a distance of $5.8 \mathrm{~cm}$ between the fixation cross and the center of the image. Participants were placed $60 \mathrm{~cm}$ from the screen using a chin-rest. This resulted in a visual angle of $5.5^{\circ}$ between 
the fixation cross and the center of the image. Participants were instructed to press the "same" button (a numeric key 1 or 2) if all faces were the same age category and the "different" button (a numeric key 2 or 1 ) if one of the faces differed in the age category from the rest. The combinations of numeric keys were assigned randomly to each participant. Participants were also asked to respond as quickly and accurately as possible. The stimulus faces remained on the screen for $6000 \mathrm{~ms}$ or until the participant responded (Figure 1).

For each set size, there were 160 trials composed of 4 blocks of 40 . There were 12 blocks in total, which were preceded by a block of 8 practice trials. The order of the blocks differed across participants.

\section{MEASUREMENT OF URINARY OXYTOCIN}

Urine was collected from participants just before the visual search task between 1400 and 1800. Participants were instructed to abstain from food and drink for $2 \mathrm{~h}$ before urine sampling. An hour before this urine sampling, participants urinated and were instructed to abstain from exercise, stressful activity (e.g., seeing exciting movies), and sleeping. Immediately after collection, urine samples were centrifuged at $4^{\circ} \mathrm{C}$ in a refrigerated centrifuge, and frozen at $-80^{\circ} \mathrm{C}$ until assayed. Urinary oxytocin concentrations were measured using radioimmunoassays (Mitsui et al., 2011). Creatinine concentrations were measured using the Jaffe reaction using 96-well microplates (3881-096, Asahi Glass Co., Japan). The plate was read at an optical density of $450 \mathrm{~nm}$ by using a microplate reader. Urinary oxytocin levels are expressed as the oxytocin to creatinine ratio. The intra-assay coefficient of variation was $4.26 \%$.

\section{RESULTS}

\section{BEHAVIORAL DATA}

Data from all participants were analyzed because error rates were lower than $10 \%$. The average error rate was $5.0 \%$. Only reaction times (RTs) to correctly identified targets and correctly rejected non-targets were included in the analyses. Before the analysis, RTs were filtered for outliers. All RTs lying more than 2 standard deviations above or below the individual mean were excluded from

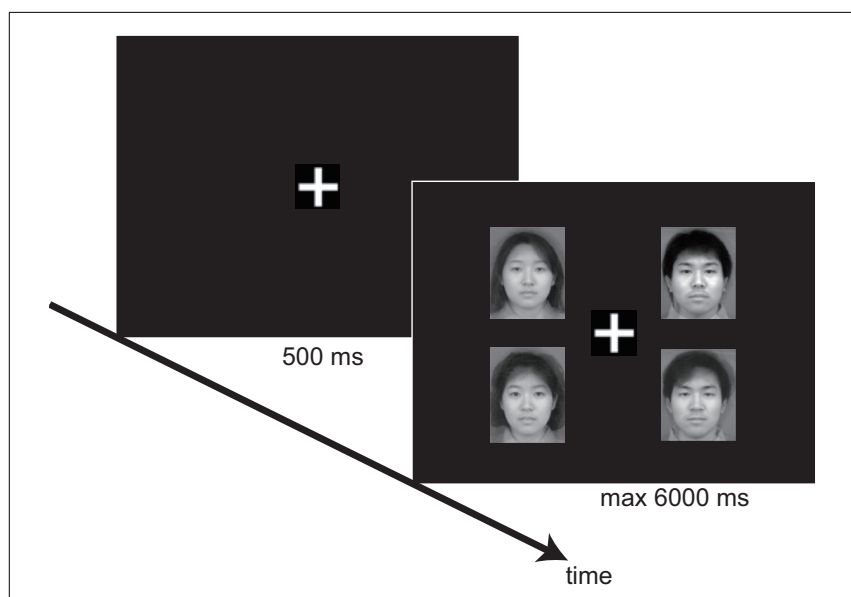

FIGURE 1 | Experimental sequence of the visual search task. the analyses. Average RTs and differences between the reaction time for infant faces and for adult faces (RTs for Infant target or distracters - RTs for Adult target or distracters) are summarized in Table 1.

\section{ASSOCIATION BETWEEN OXYTOCIN LEVELS AND PERFORMANCE IN VISUAL SEARCH}

As one participant's oxytocin concentration was extremely high $(2031.79 \mathrm{pg} / \mathrm{mg})$, we analyzed other 12 participants' data. The average oxytocin concentrations was $344.38 \mathrm{pg} / \mathrm{mg}$ (range: 117.38-753.36, $S D=196.18$ ). We calculated Speaman's correlation coefficients between RTs in the visual search task and urinary oxytocin levels (Figure 2). As shown in Figures 2A-L, the reaction time for the visual search task negatively correlated with urinary oxytocin levels; that is, the performance positively correlated with oxytocin levels. The correlation coefficients (Speamans rho) were significant for the adult target conditions of set size 3 ( $r h o=-0.587, p=0.045$, Figure 2A), and the adult condition of set size 6 and the infant condition of set size 3 in target absent condition ( $r h o=-0.706, p=0.010$; rho $=-0.587, p=0.045$, respectively, Figures 2I,J). We also calculated the statistical power of our experiments. This analysis revealed that correlations that were statistically significant had sufficient statistical power $(\sim 0.5$, Figure 2). In contrast, there were no consistent tendencies in the differences between the reaction time for infant faces and adult faces (Figures 3A-F).

\section{DISCUSSION}

Our results show that performance of the visual search task positively correlated with urinary oxytocin levels. This is the first presentation of the relationship between peripheral oxytocin levels and the performance of cognitive tasks. The response for social stimuli, both of infant and adult faces, was accelerated by high oxytocin levels. This result is consistent with many previous studies in which intranasal oxytocin administration promoted positive responses in social contexts (Kosfeld et al., 2005; Zak et al., 2007; Baumgartner et al., 2008; Petrovic et al., 2008; Theodoridou et al., 2009). However, arginine vasopressin, a neuropeptide that has a similar amino acid sequence and function as oxytocin, enhanced performance in a simple reaction time task when it was delivered by intranasal spray (Beckwith et al., 1983; Jennings

Table 1 | Average RTs (ms) and standard deviations for target-present and target-absent trials and differences between the reaction time for infant faces and that for adult faces.

\begin{tabular}{|c|c|c|c|c|c|c|}
\hline \multirow[t]{2}{*}{ Set size } & \multicolumn{2}{|c|}{3} & \multicolumn{2}{|c|}{4} & \multicolumn{2}{|c|}{6} \\
\hline & $M$ & $S D$ & $M$ & $S D$ & $M$ & $S D$ \\
\hline \multicolumn{7}{|c|}{ TARGET PRESENT } \\
\hline Adult target & 855.259 & 155.215 & 873.335 & 117.583 & 972.834 & 123.608 \\
\hline Infant target & 843.201 & 135.803 & 875.097 & 137.499 & 963.807 & 90.290 \\
\hline Difference & -12.059 & 50.304 & 1.762 & 70.636 & -9.027 & 65.710 \\
\hline \multicolumn{7}{|c|}{ TARGET ABSENT } \\
\hline Adult all & 856.670 & 149.689 & 860.864 & 120.309 & 1046.755 & 138.065 \\
\hline Infant all & 742.284 & 102.438 & 752.219 & 101.178 & 937.447 & 105.662 \\
\hline Difference & -114.386 & 68.782 & -108.645 & 64.621 & -109.308 & 86.976 \\
\hline
\end{tabular}



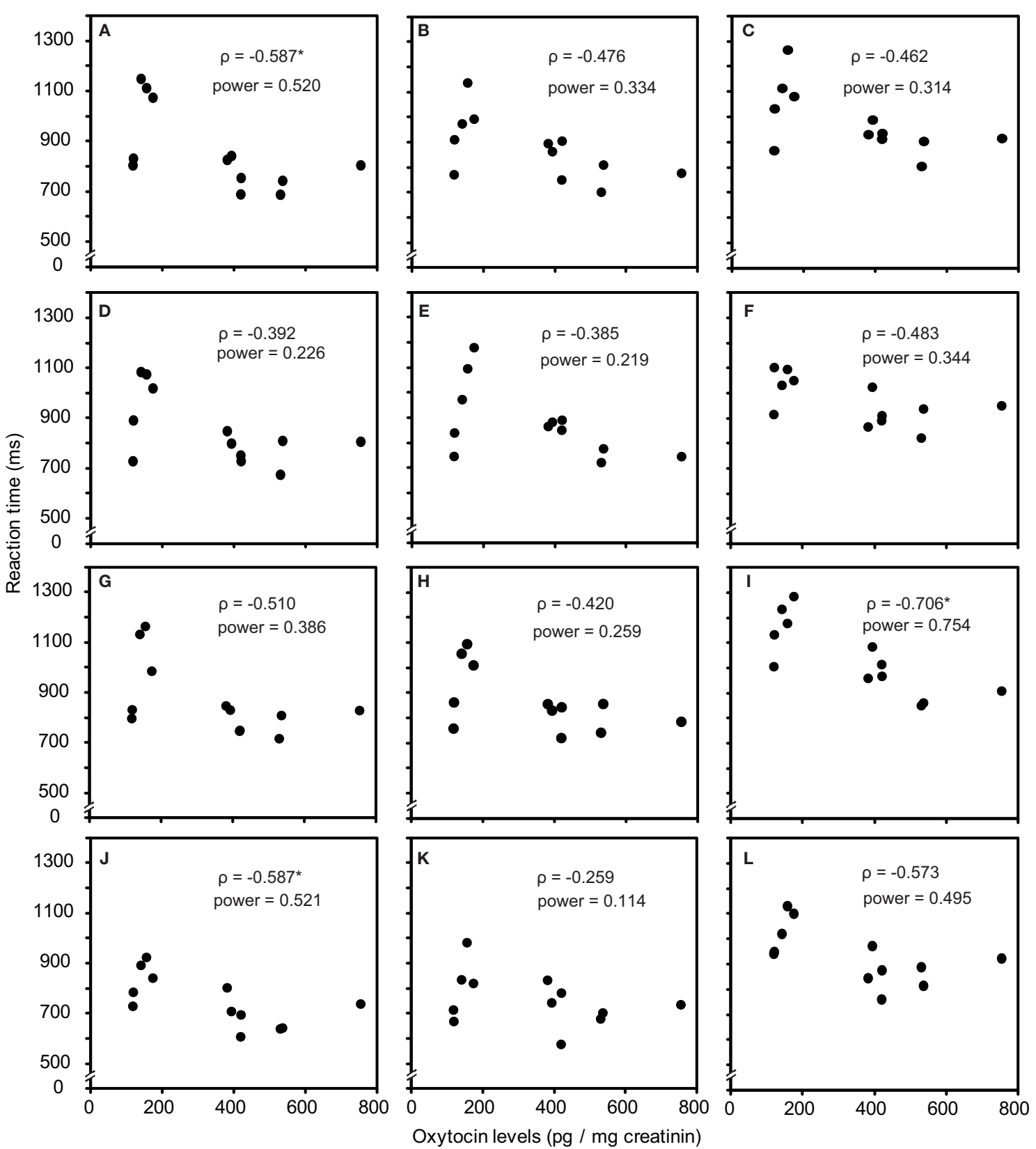

FIGURE 2 | Correlation between urinary oxytocin levels and reaction times (A-L). (A) Target present, adult target condition, set size 3; (B) Target present, adult target condition, set size 4; (C) Target present, adult target condition, set size 6; (D) Target present, infant target condition, set size 3; (E) Target present, infant target condition, set size 4; (F) Target present, infant target condition, set size 6; (G) Target absent, adult all condition, set size 3; (H) Target absent, adult all condition, set size 4; (I) Target absent, adult all condition, set size 6; (J) Target absent, infant all condition, set size 3; (K) Target absent, infant all condition, set size 4; (L) Target absent, infant all condition, set size 6 . *indicates $p<0.05$. et al., 1986). Oxytocin may also have a similar effect. In this case, the current results can easily be explained by the enhancement effect of oxytocin on the simple reaction time task. To clarify this issue, it is necessary to perform experiments using nonsocial stimuli. Collectively, although the results are preliminary due to the relatively small sample size, this is the first presentation of the relationship between endogenous oxytocin levels and the performance of cognitive tasks, which can explain the individual differences in visual search ability for social object. But we cannot find any relationship between endogenous oxytocin and visual search for infant faces in unmarried males, indicating that experience of parenting is important for enhance the visual search ability for infants.

We measured oxytocin levels in urine. There is some controversy surrounding the relationship between oxytocin levels in urine and plasma (Amico et al., 1987; Feldman et al., 2011). 


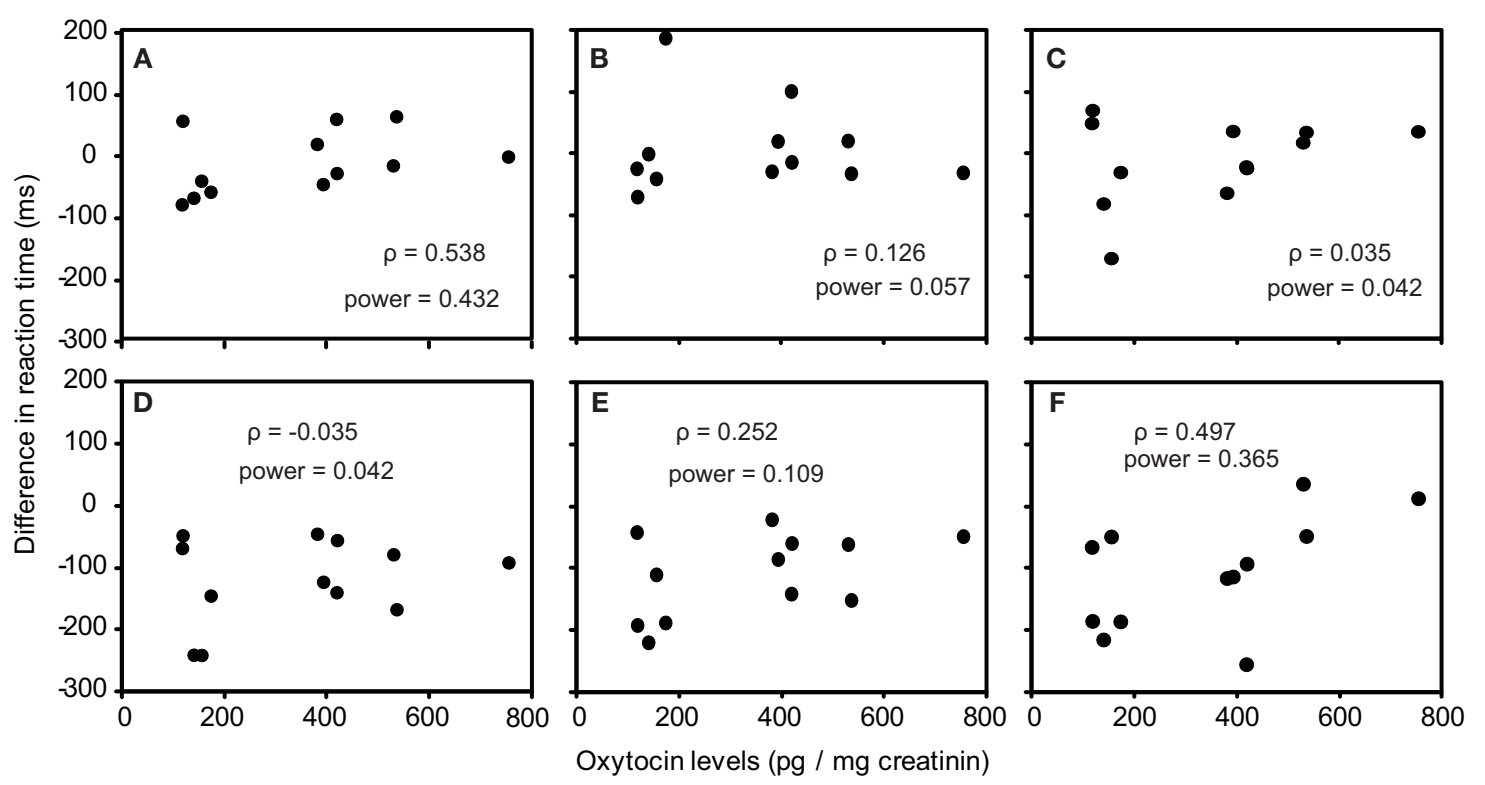

FIGURE 3 | Correlation between urinary oxytocin levels and differences between the reaction times for infant faces and adult faces (A-F). (A) Target present, set size 3; (B) Target present, set size 4; (C) Target present, set size 6; (D) Target absent, set size 3; (E) Target present, set size 4; (F) Target present, set size 6.

However, it is understandable that Feldman et al. (2011) described no correlation between urinary oxytocin and plasma oxytocin, as they measured urine and plasma at a single time point. Urinary oxytocin reflects oxytocin that has accumulated in the kidney over a period of approximately an hour. In our study, participants urinated an hour before urine sampling, and were then instructed to abstain from exercise, stressful activities (e.g., watching exciting movies), and sleeping. Therefore, our samples reflect accumulated oxytocin, excreted from the kidney over the course of an hour. In addition, we recently demonstrated a significant positive correlation between plasma oxytocin and urinary oxytocin levels in dogs (Romero et al., 2014). These results indicate that urinary oxytocin is a non-invasive biomarker that can be used to assess oxytocin activity. There is still debate over whether peripheral measures of oxytocin are related to central measures of oxytocin. However, non-invasive measures, such as urinary oxytocin, hold research and therapeutic advantages (Crockford et al., 2014). Our results improve the understanding of effects of peripheral oxytocin.

The results were somewhat different from the expected ones. The oxytocin levels positively correlated to the detected speed of infant faces as well as to that for adult faces. In addition, oxytocin levels did not relate to differences between the reaction time for infant faces and that for adult faces. A positive relationship between peripheral oxytocin and specific reaction to infants was not observed. These results seem inconsistent with the previous results showing that peripheral oxytocin levels positively correlated with paternal behavior (Gordon et al., 2010a,b,c; Feldman et al., 2011). The reason for this inconsistency may be caused by the difference in features of the participants. Almost all of the previous studies investigating the effect of oxytocin on parental behavior targeted actual parents. In contrast, the participants in our studies were non-married and non-father males. Although fathers do not have different peripheral oxytocin levels from nonfathers (Gray et al., 2007), the physical conditions of fathers, including testosterone levels, differ from those of non-fathers (Kuzawa et al., 2010; Gettler et al., 2011). These differences may explain the inconsistency between the previous studies and our study. There is the possibility that other hormonal levels interact with oxytocin and affect parent-like behavior. For example, testosterone affects expression of the oxytocin receptor in central nervous system (Arsenijevic and Tribollet, 1998) and oxytocin interacts with testosterone level and parental behavior (Weisman et al., 2014). That is, people who have different attributions, including marital status or parenthood, have different effects of oxytocin on parental behavior. Becoming fathers may change the response to infants: the effect of oxytocin on responses specialized for infants will be shown in fathers.

Our results reveal that high oxytocin levels do not always facilitate the specific reaction to infants. As Bartz et al. (2011) pointed out, the effects of oxytocin are constrained by situations and/or individualities. Therefore, it is necessary to study them by considering the features of the participants as discussed above. In addition to the different effects of oxytocin depending on the individual traits, a couple of recent studies demonstrated that oxytocin's social effects are context-dependent (Cardoso et al., 2013; Scheele et al., 2014). We should also take the difference in context into consideration when we investigate the effects of oxytocin.

\section{AUTHOR CONTRIBUTIONS}

Conceived and designed the experiments: Atsuko Saito, Hiroki Hamada, and Kazuo Hiraki. Performed the experiments: Hiroki Hamada Analyzed the data: Hiroki Hamada, Atsuko Saito, 
and Kazuo Hiraki. Hormonal measurement: Hiroki Hamada, Takefumi Kikusui, Kazutaka Mogi, Miho Nagasawa, and Shohei Mitsui and Takashi Higuchi. Prepared the manuscript: Atsuko Saito and Hiroki Hamada. Organized the research project: Kazuo Hiraki and Toshikazu Hasegawa.

\section{ACKNOWLEDGMENTS}

This work was supported by JSPS KAKENHI Grant Number 23700307 (to Atsuko Saito), 25118007 (to Takefumi Kikusui), 25118003 (to Toshikazu Hasegawa), 22240026 (to Kazuo Hiraki) and Crest JST (to Kazuo Hiraki).

\section{REFERENCES}

Amico, J. A., Ulbrecht, J. S., and Robinson, A. G. (1987). Clearance studies of oxytocin in humans using radioimmunoassay measurements of the hormone in plasma and urine. J. Clin. Endocrinol. Metab. 64, 340-345. doi: 10.1210/jcem64-2-340

Arsenijevic, Y., and Tribollet, E. (1998). Region-specific effect of testosterone on oxytocin receptor binding in the brain of the aged rat. Brain Res. 785, 167-170. doi: 10.1016/S0006-8993(97)01429-7

Bartz, J. A., Zaki, J., Bolger, N., and Ochsner, K. N. (2011). Social effects of oxytocin in humans: context and person matter. Trends Cogn. Sci. 15, 301-309. doi: 10. 1016/j.tics.2011.05.002

Baumgartner, T., Heinrichs, M., Vonlanthen, A., Fischbacher, U., and Fehr, E. (2008). Oxytocin shapes the neural circuitry of trust and trust adaptation in humans. Neuron 58, 639-650. doi: 10.1016/j.neuron.2008.04.009

Beckwith, B. E., Couk, D. I., and Till, T. S. (1983). Vasopressin analog influences the performance of males on a reaction time task. Peptides 4, 707-709. doi: 10.1016/0196-9781(83)90022-0

Bos, P. A., Panksepp, J., Bluthe, R. M., and Van Honk, J. (2012). Acute effects of steroid hormones and neuropeptides on human social-emotional behavior: a review of single administration studies. Front. Neuroendocrinol. 33, 17-35. doi: 10.1016/j.yfrne.2011.01.002

Brosch, T., Sander, D., Pourtois, G., and Scherer, K. R. (2008). Beyond fear: rapid spatial orienting toward positive emotional stimuli. Psychol. Sci. 19, 362-370. doi: $10.1111 / j .1467-9280.2008 .02094 . x$

Brosch, T., Sander, D., and Scherer, K. R. (2007). That baby caught my eye attention capture by infant faces. Emotion 7, 685-689. doi: 10.1037/1528-3542.7.3.685

Cardoso, C., Ellenbogen, M. A., Serravalle, L., and Linnen, A.-M. (2013). Stressinduced negative mood moderates the relation between oxytocin administration and trust: evidence for the tend-and-befriend response to stress? Psychoneuroendocrinology 38, 2800-2804. doi: 10.1016/j.psyneuen.2013.05.006

Churchland, P. S., and Winkielman, P. (2012). Modulating social behavior with oxytocin: how does it work? What does it mean? Horm. Behav. 61, 392-3099. doi: 10.1016/j.yhbeh.2011.12.003

Crockford, C., Deschner, T., Ziegler, T. E., and Wittig, R. M. (2014). Endogenous peripheral oxytocin measures can give insight into the dynamics of social relationships: a review. Front. Behav. Neurosci. 8:68. doi: 10.3389/fnbeh.2014.00068

Crockford, C., Wittig, R. M., Langergraber, K., Ziegler, T. E., Zuberbuhler, K., and Deschner, T. (2013). Urinary oxytocin and social bonding in related and unrelated wild chimpanzees. Proc. R. Soc. B Biol. Sci. 280, 1471-2954. doi: 10.1098/rspb.2012.2765

Donaldson, Z. R., and Young, L. J. (2008). Oxytocin, vasopressin, and the neurogenetics of sociality. Science 322, 900-904. doi: 10.1126/science.1158668

Ekman, P., and Friesen, W. V. (1976). Pictures of Facial Affect. Palo Alto, CA: Consulting Psychologists Press.

Engelmann, M., Ebner, K., Wotjak, C. T., and Landgraf, R. (1998). Endogenous oxytocin is involved in short-term olfactory memory in female rats. Behav. Brain Res. 90, 89-94. doi: 10.1016/S0166-4328(97)00084-3

Feldman, R., Gordon, I., Schneiderman, I., Weisman, O., and Zagoory-Sharon, O. (2010). Natural variations in maternal and paternal care are associated with systematic changes in oxytocin following parent-infant contact. Psychoneuroendocrinology 35, 1133-1141. doi: 10.1016/j.psyneuen.2010.01.013

Feldman, R., Gordon, I., and Zagoory-Sharon, O. (2011). Maternal and paternal plasma, salivary, and urinary oxytocin and parent-infant synchrony: considering stress and affiliation components of human bonding. Dev. Sci. 14, 752-761. doi: $10.1111 / j .1467-7687.2010 .01021 . x$
Feldman, R., Weller, A., Zagoory-Sharon, O., and Levine, A. (2007). Evidence for a neuroendocrinological foundation of human affiliation: plasma oxytocin levels across pregnancy and the postpartum period predict mother-infant bonding. Psychol. Sci. 18, 965-970. doi: 10.1111/j.1467-9280.2007.02010.x

Ferguson, J. N., Young, L. J., Hearn, E. F., Matzuk, M. M., Insel, T. R., and Winslow, J. T. (2000). Social amnesia in mice lacking the oxytocin gene. Nat. Genet. 25, 284-288. doi: 10.1038/77040

Fleming, A. S., Steiner, M., and Anderson, V. (1987). Hormonal and attitudinal correlates of maternal behavior during the early postpartum period in firsttime mothers. J. Reprod. Infant Psyc. 5, 193-205. doi: 10.1080/02646838708 403495

Fries, A. B., Ziegler, T. E., Kurian, J. R., Jacoris, S., and Pollak, S. D. (2005). Early experience in humans is associated with changes in neuropeptides critical for regulating social behavior. Proc. Natl. Acad. Sci. U.S.A. 102, 17237-17240. doi: 10.1073/pnas.0504767102

Gettler, L. T., McDade, T. W., Feranil, A. B., and Kuzawa, C. W. (2011). Longitudinal evidence that fatherhood decreases testosterone in human males. Proc. Natl. Acad. Sci. U.S.A. 108, 16194-16199. doi: 10.1073/pnas.1105403108

Gordon, I., Zagoory-Sharon, O., Leckman, J. F., and Feldman, R. (2010a). Oxytocin and the development of parenting in humans. Biol. Psychiatry 68, 377-382. doi 10.1016/j.biopsych.2010.02.005

Gordon, I., Zagoory-Sharon, O., Leckman, J. F., and Feldman, R. (2010b). Oxytocin, cortisol, and triadic family interactions. Physiol. Behav. 101, 679-684. doi: 10.1016/j.physbeh.2010.08.008

Gordon, I., Zagoory-Sharon, O., Leckman, J. F., and Feldman, R. (2010c). Prolactin, Oxytocin, and the development of paternal behavior across the first six months of fatherhood. Horm. Behav. 58, 513-518. doi: 10.1016/j.yhbeh.2010.04.007

Gray, P. B., Parkin, J. C., and Samms-Vaughan, M. E. (2007). Hormonal correlates of human paternal interactions: a hospital-based investigation in urban Jamaica. Horm. Behav. 52, 499-507. doi: 10.1016/j.yhbeh.2007.07.005

Guastella, A. J., and Macleod, C. (2012). A critical review of the influence of oxytocin nasal spray on social cognition in humans: evidence and future directions. Horm. Behav. 61, 410-418. doi: 10.1016/j.yhbeh.2012.01.002

Insel, T. R., and Hulihan, T. J. (1995). A gender-specific mechanism for pair bonding: oxytocin and partner preference formation in monogamous voles. Behav. Neurosci. 109, 782-789. doi: 10.1037/0735-7044.109.4.782

Jennings, J. R., Nebes, R. D., and Reynolds, C. F. 3rd. (1986). Vasopressin peptide (DDAVP) may narrow the focus of attention in normal elderly. Psychiatry Res. 17, 31-39. doi: 10.1016/0165-1781(86)90039-9

Kosfeld, M., Heinrichs, M., Zak, P. J., Fischbacher, U., and Fehr, E. (2005). Oxytocin increases trust in humans. Nature 435, 673-676. doi: 10.1038/nature03701

Kozorovitskiy, Y., Hughes, M., Lee, K., and Gould, E. (2006). Fatherhood affects dendritic spines and vasopressin V1a receptors in the primate prefrontal cortex. Nat. Neurosci. 9, 1094-1095. doi: 10.1038/nn1753

Kuzawa, C. W., Gettler, L. T., Huang, Y. Y., and McDade, T. W. (2010). Mothers have lower testosterone than non-mothers: evidence from the Philippines. Horm. Behav. 57, 441-447. doi: 10.1016/j.yhbeh.2010.01.014

Mitsui, S., Yamamoto, M., Nagasawa, M., Mogi, K., Kikusui, T., Ohtani, N., et al. (2011). Urinary oxytocin as a noninvasive biomarker of positive emotion in dogs. Horm. Behav. 60, 239-243. doi: 10.1016/j.yhbeh.2011.05.012

Muller, H. J., and Krummenacher, J. (2006). Visual search and selective attention. Vis. Cogn. 14, 389-410. doi: 10.1080/13506280500527676

Nagasawa, M., Kikusui, T., Onaka, T., and Ohta, M. (2009). Dog's gaze at its owner increases owner's urinary oxytocin during social interaction. Horm. Behav. 55 , 434-441. doi: 10.1016/j.yhbeh.2008.12.002

Nittono, H., Fukushima, M., Yano, A., and Moriya, H. (2012). The power of kawaii: viewing cute images promotes a careful behavior and narrows attentional focus. PLoS ONE 7:e46362. doi: 10.1371/journal.pone.0046362

Pedersen, C. A., Ascher, J. A., Monroe, Y. L., and Prange, A. J. Jr. (1982). Oxytocin induces maternal behavior in virgin female rats. Science 216, 648-650. doi: 10.1126/science.7071605

Pedersen, C. A., and Prange, A. J. Jr. (1979). Induction of maternal behavior in virgin rats after intracerebroventricular administration of oxytocin. Proc. Natl. Acad. Sci. U.S.A. 76, 6661-6665. doi: 10.1073/pnas.76.12.6661

Petrovic, P., Kalisch, R., Singer, T., and Dolan, R. J. (2008). Oxytocin attenuates affective evaluations of conditioned faces and amygdala activity. J. Neurosci. 28, 6607-6615. doi: 10.1523/JNEUROSCI.4572-07.2008

Riem, M. M., Bakermans-Kranenburg, M. J., Pieper, S., Tops, M., Boksem, M. A., Vermeiren, R. R., et al. (2011). Oxytocin modulates amygdala, insula, and 
inferior frontal gyrus responses to infant crying: a randomized controlled trial. Biol. Psychiatry. 70, 291-297. doi: 10.1016/j.biopsych.2011.02.006

Romero, T., Nagasawa, M., Mogi, K., Hasegawa, T., and Kikusui, T. (2014). Oxytocin promotes social bonding in dogs. Proc. Natl. Acad. Sci. U.S.A. 111, 9085-9090. doi: 10.1073/pnas.1322868111

Rupp, H. A., James, T. W., Ketterson, E. D., Sengelaub, D. R., Ditzen, B., and Heiman, J. R. (2012). Amygdala response to negative images in postpartum vs nulliparous women and intranasal oxytocin. Soc. Cogn. Affect. Neurosci. 9, 48-54. doi: 10.1093/scan/nss 100

Salonia, A., Nappi, R. E., Pontillo, M., Daverio, R., Smeraldi, A., Briganti, A., et al. (2005). Menstrual cycle-related changes in plasma oxytocin are relevant to normal sexual function in healthy women. Horm. Behav. 47, 164-169. doi: 10.1016/j.yhbeh.2004.10.002

Scheele, D., Kendrick, K. M., Khouri, C., Kretzer, E., Schläpfer, T. E., StoffelWagner, B., et al. (2014). An oxytocin-induced facilitation of neural and emotional responses to social touch correlates inversely with autism traits. Neuropsychopharmacology 39, 2078-2085. doi: 10.1038/npp.2014.78

Seltzer, L. J., Ziegler, T. E., and Pollak, S. D. (2010). Social vocalizations can release oxytocin in humans. Proc. R. Soc. B Biol. Sci. 277, 2661-2666. doi: 10.1098/rspb.2010.0567

Snowdon, C. T., Pieper, B. A., Boe, C. Y., Cronin, K. A., Kurian, A. V., and Ziegler, T. E. (2010). Variation in oxytocin is related to variation in affiliative behavior in monogamous, pairbonded tamarins. Horm. Behav. 58, 614-618. doi: 10.1016/j.yhbeh.2010.06.014

Swain, J. E. (2008). Baby stimuli and the parent brain: functional neuroimaging of the neural substrates of parent-infant attachment. Psychiatry 5, 28-36.

Strathearn, L., Fonagy, P., Amico, J., and Montague, P. R. (2009). Adult attachment predicts maternal brain and oxytocin response to infant cues. Neuropsychopharmacology 34, 2655-2666. doi: 10.1038/npp.2009.103

Theodoridou, A., Rowe, A. C., Penton-Voak, I. S., and Rogers, P. J. (2009). Oxytocin and social perception: oxytocin increases perceived facial trustworthiness and attractiveness. Horm. Behav. 56, 128-132. doi: 10.1016/j.yhbeh.2009.03.019

Van Ijzendoorn, M. H., and Bakermans-Kranenburg, M. J. (2012). A sniff of trust: meta-analysis of the effects of intranasal oxytocin administration on face recognition, trust to in-group, and trust to out-group. Psychoneuroendocrinology 37, 438-443. doi: 10.1016/j.psyneuen.2011.07.008

Weisman, O., Zagoory-Sharon, O., and Feldman, R. (2012). Oxytocin administration to parent enhances infant physiological and behavioral readiness for social engagement. Biol. Psychiatry 72, 982-989. doi: 10.1016/j.biopsych.2012.06.011
Weisman, O., Zagoory-Sharon, O., and Feldman, R. (2014). Oxytocin administration, salivary testosterone, and father-infant social behavior. Prog. Neuropsychopharmacol. Biol. Psychiatry 49, 47-52. doi: 10.1016/j.pnpbp.2013. 11.006

Williams, J. R., Insel, T. R., Harbaugh, C. R., and Carter, C. S. (1994). Oxytocin administered centrally facilitates formation of a partner preference in female prairie voles (Microtus ochrogaster). J. Neuroendocrinol. 6, 247-250. doi: 10.1111/j.1365-2826.1994.tb00579.x

Wittig, R. M., Crockford, C., Deschner, T., Langergraber, K. E., Ziegler, T. E., and Zuberbuhler, K. (2014). Food sharing is linked to urinary oxytocin levels and bonding in related and unrelated wild chimpanzees. Proc. R. Soc. B Biol. Sci. 281:20133096. doi: 10.1098/rspb.2013.3096

Zak, P. J., Kurzban, R., and Matzner, W. T. (2005). Oxytocin is associated with human trustworthiness. Horm. Behav. 48, 522-527. doi: 10.1016/j.yhbeh.2005. 07.009

Zak, P. J., Stanton, A. A., and Ahmadi, S. (2007). Oxytocin increases generosity in humans. PLoS ONE 2:e1128. doi: 10.1371/journal.pone.0001128

Zink, C. F., and Meyer-Lindenberg, A. (2012). Human neuroimaging of oxytocin and vasopressin in social cognition. Horm. Behav. 61, 400-409. doi: 10.1016/j. yhbeh.2012.01.016

Conflict of Interest Statement: The authors declare that the research was conducted in the absence of any commercial or financial relationships that could be construed as a potential conflict of interest.

Received: 26 February 2014; accepted: 04 July 2014; published online: 29 July 2014. Citation: Saito A, Hamada H, Kikusui T, Mogi K, Nagasawa M, Mitsui S, Higuchi T, Hasegawa T and Hiraki $K$ (2014) Urinary oxytocin positively correlates with performance in facial visual search in unmarried males, without specific reaction to infant face. Front. Neurosci. 8:217. doi: 10.3389/fnins.2014.00217

This article was submitted to Neuroendocrine Science, a section of the journal Frontiers in Neuroscience.

Copyright (c) 2014 Saito, Hamada, Kikusui, Mogi, Nagasawa, Mitsui, Higuchi, Hasegawa and Hiraki. This is an open-access article distributed under the terms of the Creative Commons Attribution License (CC BY). The use, distribution or reproduction in other forums is permitted, provided the original author(s) or licensor are credited and that the original publication in this journal is cited, in accordance with accepted academic practice. No use, distribution or reproduction is permitted which does not comply with these terms. 